\title{
Socio-Psychological Resilience of the Russian Enterprises Personnel: Basic Types and Social Influence
}

\author{
L. Zakharova, \\ E. Korobeynikova, \\ Nizhny Novgorod State University
}

\begin{abstract}
Objective: To reveal socio-psychological features of the personnel of Russian enterprises with different levels of resilience. The theoretical framework: The theories by T.Parsons, K.Cameron and R.Quinn. Empirical basis: Research period: 1999-2015. Subjects: not less than 20 senior managers, 120 mid-level managers, 360 lower-level employees annually. Results. Organizational conditions of successful high-technology enterprises $(\mathrm{H}-\mathrm{TE})$ and ordinary enterprises (OE) with long time modernization difficulties are characterized by specific types of organizational culture, leadership and managerial interaction. The staff of these enterprises differ in the following parameters: susceptibility to organizational stress, value priorities of organization development, balance of work and day-to-day motivation of managers and staff, levels of responsibility and transparency of their decisions, mutual confidence, susceptibility to pre-reform stereotypes, balance of value and instrumental components in determination of conflict behaviour, ratio of the main strategies of conflict behaviour and stress coping. Sets of these parameters form two types of psychological resilience (PsR): perspective at the H-TE and situational at the OE. Perspective psychological resilience is a factor of company development, is of an open form and is characterized by stress resistance in the conditions of change and personnel readiness towards innovation acceptance. Conclusion. The H-TE staff is characterized by forward-looking (strategic) type of PsR, while the OE staff - by situational (tactical) type. Ethical implications. Ensuring the right PsR of the staff in enterprises by managing organizational conditions can enhance the resilience both enterprises and traditional society.
\end{abstract}

Keywords: socio-psychological resilience, organizational conditions, staff, organizational culture, leadership, managerial interaction. 